Check for updates

Cite this: RSC Adv., 2019, 9, 6278

Received 20th January 2019

Accepted 15th February 2019

DOI: $10.1039 / c 9 r a 00500 e$

rsc.li/rsc-advances

\section{Gamma ray assisted modification of carbon quantum dot/polyurethane nanocomposites: structural, mechanical and photocatalytic study $\dagger$}

\author{
Milica Budimir, (D) *ab Zoran Marković, ${ }^{a}$ Dragana Jovanović, ${ }^{a}$ Miloš Vujisić, \\ Matej Mičušik, ${ }^{c}$ Martin Danko, ${ }^{c}$ Angela Kleinová, ${ }^{c}$ Helena Švajdlenková, (D) ${ }^{c}$ \\ Zdeno Špitalskýc and Biljana Todorović Marković (D) a
}

\begin{abstract}
In recent years, water pollution and contamination had become a major threat to the ecosystem. However, the use of nanostructured materials has been proven as a very promising approach in the treatment of polluted water. The present study reports the results of the gamma ray-assisted modification of hydrophobic carbon quantum dot (hCQD)/polyurethane nanocomposites for photocatalytic degradation of organic dyes. Different characterization methods were applied to investigate the influence of the different doses of gamma irradiation (1,10 and $200 \mathrm{kGy}$ ) on the physical and chemical properties of nanocomposites (morphology, chemical content, mechanical properties, wettability, and potential for singlet oxygen generation). Surface morphology and mechanical properties analyses showed that gamma rays induced insignificant changes in the structure of nanocomposites, but the potential for singlet oxygen generation increased significantly. Here we also explore, in detail, the photocatalytic properties of gamma-ray modified hCQDs/polyurethane nanocomposites. UV-vis analysis showed that the removal efficiency of the rose bengal dye reached up to $97 \%$ for the nanocomposite irradiated with the dose of 200 kGy.
\end{abstract}

\section{Introduction}

Our ecosystem is becoming more endangered every year due to the growing urbanization and industrialization. Many industries are responsible for water pollution, and one of the main sources of pollution is the dyeing industry. These contaminants have a vast impact on aquatic flora and fauna and due to their persistence, they can remain in the environment for a very long time. Rose Bengal (RB) is a commonly used dye in the textile and printing industries. However, this dye belongs to the group of xanthene dyes, which are reported to be cytotoxic, genotoxic and cytostatic., ${ }^{1,2}$ Therefore, the removal of the RB dye from wastewater is very important. Recently, nanotechnology has offered many innovations in the fields of science and technology and nowadays it provides a potential substitution of the conventional water treatment procedures. Various studies have already proved the capacity and potential of nanomaterials in

${ }^{a}$ Vinča Institute of Nuclear Sciences, University of Belgrade, Mike Petrovića Alasa 12-14, 11001 Belgrade, Serbia. E-mail: budimir@vinca.rs

${ }^{b}$ School of Electrical Engineering, University of Belgrade, Bulevar kralja Aleksandra 73 , 11000 Belgrade, Serbia

'Polymer Institute, Slovak Academy of Sciences, Dúbravská cestá 9, 84541 Bratislava, Slovakia

$\dagger$ Electronic supplementary information (ESI) available. See DOI: 10.1039/c9ra00500e water and wastewater treatment, including the areas of adsorption, catalytic oxidation, nanostructured membranes, disinfection and sensors. ${ }^{3-6}$ Numerous methods have been developed for the removal of synthetic dyes from water and waste-water, including the adsorption by inorganic or organic matrices, decolorization by photocatalysis or by oxidation processes, microbiological or enzymatic decomposition, etc. ${ }^{7,8}$

The majority of nanomaterials used in water treatment are present as nanoparticles. However, when concerning the largescale application of nanoparticles in water treatment, they must overcome some drawbacks, such as aggregation, leakage and difficult separation from the contact water, potential harmful effect on the environment and human health. The development of nanocomposites has proved to be an advantageous and promising approach that overcomes these flaws. Nanocomposites are commonly fabricated by loading desired nanoparticles onto various supporting materials, such as polymers or membranes. ${ }^{9,10}$ Nanocomposites generally take advantages of both the hosts and impregnated nanoparticles. In addition, nanocomposites could control the release of nanoparticles into the environment and enhance the compatibility of nanotechnology with existing infrastructures. ${ }^{\mathbf{6}, 11}$

Carbon quantum dots (CQDs) are a class of zerodimensional nano-carbons, which have drawn considerable attention due to their unique properties. ${ }^{12,13}$ The main features of CQDs: they are quasi-spherical nanoparticles with sizes 
below $10 \mathrm{~nm}$; they have excellent chemical stability and water solubility, resistance to photobleaching and they display multicolour emission in the visible region. ${ }^{14}$ CQDs are also able to generate reactive oxygen species (ROS) upon photo-excitation, which makes them good candidates for photodynamic therapy. ${ }^{\mathbf{1 4 , 1 5}}$ Another important application of CQDs is in bioimaging. ${ }^{16-18}$ Beside the mentioned properties, CQDs can be used as photocatalysts due to their good photo-induced electron transfer ability and light absorption. However, photocatalytic application of CQDs has some drawbacks, but they can be reduced by certain modification of CQDs including doping, surface inflection or design of metal or semiconductor/CQDs composites. ${ }^{19}$

Gamma irradiation is a form of electromagnetic radiation, which involves emitting of high-energy photons of very short wavelengths. Those photons can create ionization (electronic disruptions) throughout the product. Therefore, gamma irradiation is used for many different purposes, such as sterilization of medical devices, radiotherapy, microbial decontamination of food, cosmetics and their packaging, but also for the modification of different materials. During interactions of high-energy photons and different materials such as polymers or carbon nanomaterials, many complex processes appear, such as structural modifications (oxidation, reduction, cutting), bond breaking, free radical formation, crosslinking, chain scissions etc. $^{20}$ The motivation to use gamma irradiation as a modification method in the presented research were the results obtained previously in our group. These results showed different effects of gamma irradiation on graphene quantum dots depending on the irradiation dose, and one of the most important results was an improved singlet oxygen generation ability upon photoexcitation. ${ }^{21}$

In this paper, we will present a nanocomposite that consists of hydrophobic carbon quantum dots (hCQDs) incorporated in the polymer matrix. The nanocomposite was modified by different doses of gamma irradiation. Previously, a group of authors presented interesting properties of such a nanocomposite, ${ }^{22}$ without any modifications. Therefore, we decided to explore the modification possibilities by gamma irradiation, with the possible improvement of certain properties of the nanocomposite. For the formation of hCQDs we used the bottom-up method. Later, the hCQDs dispersed in toluene were incorporated inside the polyurethane matrix by the swellencapsulation-shrink method. Finally, different doses of gamma irradiation (1, 10 and $200 \mathrm{kGy}$ ) were applied and we analysed the properties of the as-prepared nanocomposites. We have investigated how the gamma irradiation dose affected physical and chemical properties of the nanocomposites, as well as their photocatalytic activity upon the degradation of rose bengal dye.

\section{Experimental}

\subsection{Materials}

Commercially available medical grade transparent polyurethane (PU) donated by American Polyfilm with a thickness of $1.0 \mathrm{~mm}$ was used. Polyoxyethylene-polyoxypropylene- polyoxyethylene Pluronic 68 was purchased from Interchim, France, and phosphoric acid and toluene were purchased from Sigma Aldrich, Germany and used as received.

\subsection{Synthesis of hCQDs}

The synthesis of hydrophobic carbon quantum dots (hCQDs) was performed following the previously reported procedure. ${ }^{23}$ First, $1 \mathrm{~g}$ of polyoxyethylene-polyoxypropylene-polyoxyethylene Pluronic (PF-68) was dissolved in $100 \mathrm{~mL}$ of water for about $15 \mathrm{~min}$, in an ultrasonic water bath. Then $200 \mathrm{~mL}$ of phosphoric acid was added, and the mixture was stirred in order to produce a homogeneous reaction mixture. The reaction mixture was then heated on a magnetic stirrer at $250{ }^{\circ} \mathrm{C}$ for $145 \mathrm{~min}$ until the solution became brown. The mixture was cooled down to room temperature, and then $250 \mathrm{~mL}$ of water was added and stirred for another $2 \mathrm{~h}$ until it obtained a brownish black coloured precipitate. Finally, $300 \mathrm{~mL}$ of toluene was added to the solution. The reaction mixture was stirred overnight at the room temperature until toluene obtained a yellow colour. Finally, the organic phase was decanted and then filtered using a vacuum pump, through the hydrophobic filters with the pore sizes of 0.2 and $0.1 \mu \mathrm{m}$. The obtained product was a colloid of hydrophobic carbon quantum dots - hCQDs. The concentration of hCQDs was $1 \mathrm{mg} \mathrm{mL} \mathrm{m}^{-1}$.

\subsection{Preparation of hydrophobic carbon quantum dots/ polyurethane nanocomposite (hCQD-PU)}

Transparent PU was dipped in the solution of hCQDs with a concentration of $1 \mathrm{mg} \mathrm{mL}^{-1}$. The encapsulation of hCQDs into the PU was done by the swelling method at ambient temperature during $48 \mathrm{~h}$. The obtained nanocomposite hCQD-PU was dried in the vacuum furnace for $12 \mathrm{~h}$ at $80{ }^{\circ} \mathrm{C}$, in order to evaporate all the remaining toluene. ${ }^{22}$ Samples were then exposed to the source of gamma irradiation absorbing the doses of 1,10 and $200 \mathrm{kGy}$ (in further text, samples are labelled as hCQD-PU, hCQD-PU-1, hCQD-PU-10 and hCQD-PU-200). As a reference (control) we used hCQD-PU before gamma irradiation and hCQDs dispersion in toluene which was also exposed to gamma irradiation at the same doses applied.

\subsection{Photocatalytic experiments}

For the photocatalytic activity study, the hCQD-PU, hCQD-PU-1, hCQD-PU-10 and hCQD-PU-200 samples were dipped in Rose Bengal (RB, $0.03 \mathrm{mM}$ ) water solution and exposed to the blue visible lamp irradiation ( $470 \mathrm{~nm}, 3 \mathrm{~W}$ ). Each sample had surface area of $1 \times 1 \mathrm{~cm}^{2}$ and they were dipped in vials with $4 \mathrm{~mL}$ of $\mathrm{RB}$ solution. The dye degradation rate was studied by changing the irradiation time (30, 60, 120, 180 and $720 \mathrm{~min})$ and performing the UV-vis spectroscopy after each measurement.

\subsection{Characterization of the hCQDs and hCQDs/PU nanocomposites}

The samples were irradiated by gamma-ray flux from ${ }^{60} \mathrm{Co}$ nuclide with the photon energy of $1.3 \mathrm{MeV}$ (Centre of 
Irradiation, Vinca Institute of Nuclear Sciences) at a dose rate of $13 \mathrm{kGy} \mathrm{h}^{-1}$.

Fourier transform infrared (FTIR) spectroscopy and Xphotoelectron spectroscopy (XPS) were used to determine the chemical composition of the gamma-irradiated hCQD-PU nanocomposites. XPS measurement was performed on Thermo Scientific K-Alpha XPS system using monochromatic Al $\mathrm{K} \alpha \mathrm{X}$-ray source ${ }^{23}$ and FTIR was performed on a Nicolet 8700 spectrometer at ambient temperature (the spectral range from 400 to $4000 \mathrm{~cm}^{-1}$, the spectral resolution of $4 \mathrm{~cm}^{-1}$ ).

UV-vis spectra of gamma-irradiated hCQD-PU nanocomposites before and after immersion in water were measured on a Shimadzu UV-vis-NIR SolidSpec-3700 spectrophotometer to check the potential leaching of hCQDs.

The contact angle measurements of the gamma-irradiated hCQD-PU nanocomposites were conducted by the Surface Energy Evaluation System (SEE System; Advex Instruments, Czech Republic) and the software from this system has been used for further analysis. The contact angle was measured using $2 \mu \mathrm{L}$ of deionized water. The accuracy is $\pm 2^{\circ}$, and all measurements were performed in triplicates in the ambient atmosphere and at room temperature.

Dynamic mechanical analysis (DMA) of neat PU and gamma irradiated hCQDs/PU samples was performed using a TA Instrument dynamic mechanical analyzer DMA Q800. The temperature range was from $-100{ }^{\circ} \mathrm{C}$ to $100{ }^{\circ} \mathrm{C}$. The samples of uniform shapes were measured in the module tensile multifrequency strain at $10 \mathrm{~Hz}$, strain amplitude of $5 \mu \mathrm{m}$ and heating rate $2{ }^{\circ} \mathrm{C} \min ^{-1}$. All measurements were carried out without and with irradiation by blue light (BL) during $24 \mathrm{~h}$.

Surface morphology of the gamma-irradiated hCQD-PU nanocomposites was recorded by atomic force microscope AFM. Measurements were performed using a Quesant microscope (Ambios Technology, USA), and its own software. The AFM measurements were performed in air, using silicone Tshaped cantilever with a spring constant of $40 \mathrm{~N} \mathrm{~m}^{-1}$. All images were obtained at $1 \mathrm{~Hz}$, with $512 \times 512$ image resolution, over different square areas. Root-mean-square roughness-RMS of hCQDs-PU nanocomposites was determined by Gwyddion software. $^{24}$

Photoluminescence (PL) measurements of the gammairradiated hCQD-PU nanocomposites were performed on an RF-5301PC (Shimadzu, Japan) Spectro fluorophotometer, at excitation wavelengths between 320 and $480 \mathrm{~nm}$.

Electron paramagnetic resonance (EPR) is a technique used to study chemical species with unpaired electrons. EPR was used to determine the singlet oxygen generation of different hCQD-PU nanocomposites. With ${ }^{1} \mathrm{O}_{2}, 2,2,6,6$-tetramethylpiperidine (TEMP) molecules quickly react and form a stable, EPR active product, TEMP- ${ }^{1} \mathrm{O}_{2}$ (TEMPO). The concentration of TEMP was $2 \%$ wt. The EPR experiments were performed at ambient temperature using a Varian E-line spectrometer. The spectrometer operated at a frequency of $9.5 \mathrm{GHz}$. The gamma irradiated hCQD-PU nanocomposites and PU control were dipped in TEMP solution. All samples were treated in the following conditions: dark and exposed to $\mathrm{BL}$, for $12 \mathrm{~h}$, respectively. The wavelength of BL was $470 \mathrm{~nm}$ ( $3 \mathrm{~W}$, V-TAC, Bulgaria).

\section{Results and discussion}

\subsection{Fourier transform infrared - FTIR}

FTIR spectroscopy was used to assess the presence of different functional groups (Fig. 1). FTIR spectra suggested that all the observed gamma irradiated hCQD-PU nanocomposites were comprised of the absorption peak at $3300 \mathrm{~cm}^{-1}$ attributed to O-H vibration mode. The peaks at 2919, 2852 and $2797 \mathrm{~cm}^{-1}$ belong to $\mathrm{C}-\mathrm{H}$ stretching vibrations. The peaks at 1729, 1698 and $1635 \mathrm{~cm}^{-1}$ belong to $\mathrm{C}=\mathrm{O}$ bonds, while the peak at $1417 \mathrm{~cm}^{-1}$ belongs to aromatic $\mathrm{C}=\mathrm{C}$ bonds which are connected with the presence of $\mathrm{sp}^{2}$-hybridized graphitic cores. ${ }^{25}$ The strong peaks at 1220 and $1080 \mathrm{~cm}^{-1}$ are attributed to $\mathrm{C}-\mathrm{O}-\mathrm{C}$ and $\mathrm{C}-\mathrm{O}$ bonds, respectively. By analyzing the FTIR results it was concluded that the same bands were identified in hCQD-PU nanocomposites for all the different doses of gamma irradiation. Also, comparing to the FTIR results of hCQD-PU sample without gamma irradiation, from the ref. 22 we can conclude that the same bands can be identified. Therefore, from this point of view, we can conclude that gamma irradiation, at any examined irradiation dose, had no influence on the overall presence of functional groups of hCQD-PU nanocomposites.

\subsection{X-ray photoelectron spectroscopy (XPS)}

XPS analysis was carried out to explore and compare the elemental composition of gamma-irradiated hCQD-PU nanocomposites (Fig. 2). The XPS elemental survey spectrum revealed that these nanocomposites were composed of the following elements: $\mathrm{C}, \mathrm{O}$ and $\mathrm{N}$ (Table $\mathrm{S} 1 \dagger$ ). By fitting $\mathrm{C} 1 \mathrm{~s}$ and $\mathrm{O}$ $1 \mathrm{~s}$ the content of the characteristic bonds in hCQD-PU-1, hCQDPU-10 and hCQD-PU-200 were detected and compared. The XPS analysis showed that the content of oxygen is almost doubled in the sample hCQD-PU-200, compared to the sample hCQD-PU-10 (Table $\mathrm{S} 1 \dagger$ ).

According to the XPS results presented in Fig. 2 and Table $\mathrm{S} 1, \dagger$ the percentage of $\mathrm{sp}^{2}$ bonds increased with the increase of the dose of gamma irradiation (from $28.9 \%$ for hCQD-PU-1 to $49.2 \%$ for hCQD-PU-200). On the contrary, the percentage of $\mathrm{sp}^{3}$

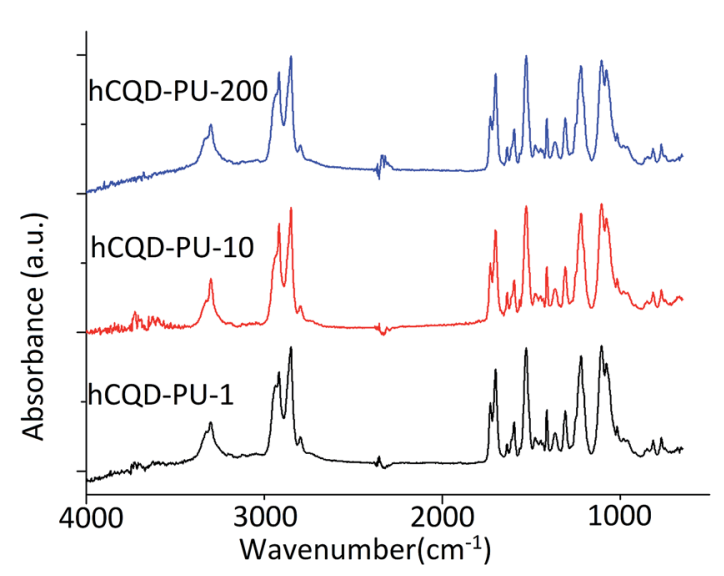

Fig. 1 FTIR spectra of hCQD-PU-1, hCQD-PU-10 and hCQD-PU-200 samples. 
a)

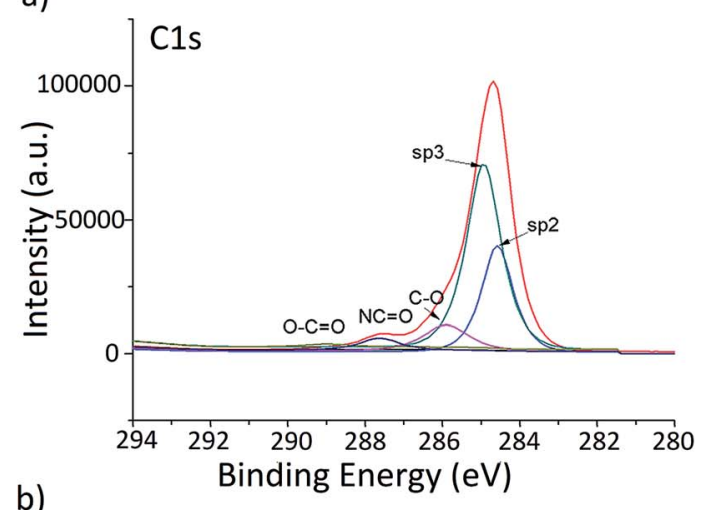

b)

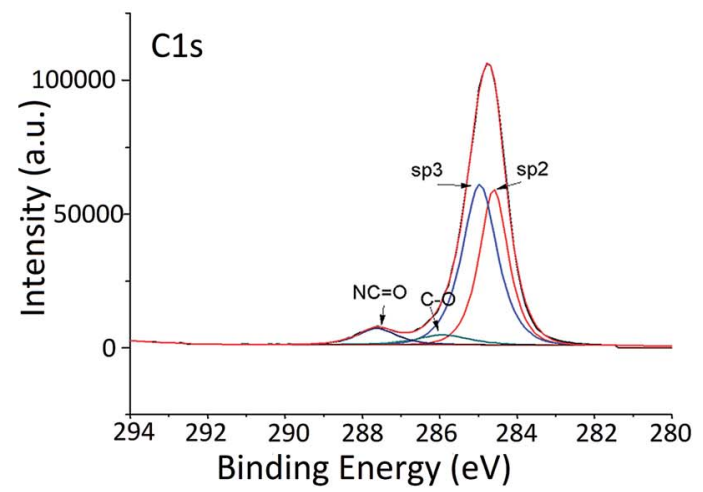

c)

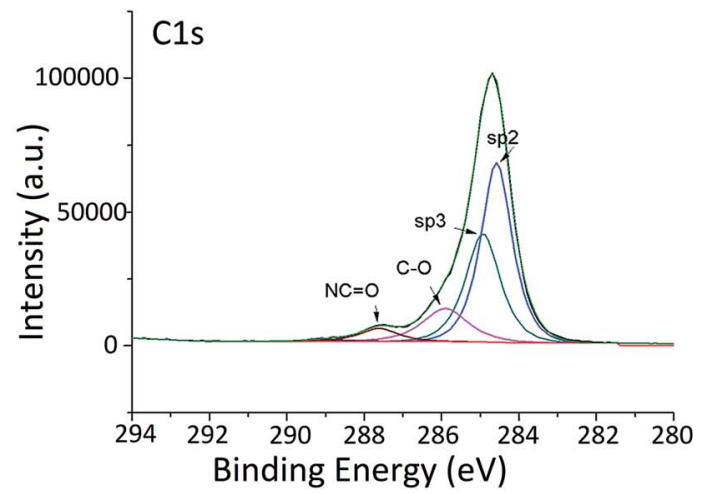

Fig. 2 XPS C 1s spectra of (a) hCQD-PU-1; (b) hCQD-PU-10 and (c) hCQD-PU-200 nanocomposites.

bonds decreased with the increase of the gamma irradiation dose (from $55.2 \%$ for hCQD-PU-1 to $32.8 \%$ for hCQD-PU-200), implying that the structure of hCQDs inside the PU polymer matrix was becoming more arranged. ${ }^{26}$

Obtained results are in accordance with the PL results, which demonstrated that the hCQD-PU-200 sample had the highest photoluminescence (Section 3.8). However, the content of oxygenated bonds was the lowest for the hCQD-PU10 sample. The contribution of $\mathrm{C}-\mathrm{O}$ bonds first decreased from $10.2 \%$ for hCQD-PU-1 to $4.4 \%$ for the hCQD-PU-10 sample and then increased again to $13 \%$ for the hCQD-PU200 sample. This result is in accordance with the EPR results which have shown that hCQD-PU-10 had the highest production of singlet oxygen.
The presence of $\mathrm{C}=\mathrm{O}$ bonds in the FTIR and the absence of the same bonds in XPS spectra suggest that hCQDs are not formed on the surface of the polymer matrix but incorporated inside.

\subsection{Leaching experiments}

To exclude the existence of leaching from the gamma-irradiated hCQD-PU nanocomposites, the samples $\left(1 \times 1 \mathrm{~cm}^{2}\right)$ were dipped in $20 \mathrm{~mL}$ of Milly-Q water for $24 \mathrm{~h}$. Recorded UV-vis spectra of water aliquots in which hCQD-PU nanocomposites were immersed, were compared with the UV-vis absorbance spectra of gamma-irradiated toluene dispersions of hCQDs (hCQD-1, hCQD-10 and hCQD-200). UV-vis spectrophotometry was used to explore the existence of leaching of the hCQD nanocomposites. From Fig. S1a $\uparrow$ the absorbance spectra of the gamma-irradiated hCQD dispersions can be observed, together with the absorbance spectra of the aliquots of water in which hCQD-PU-1, hCQD-PU-10 and hCQD-PU-200 nanocomposites were immersed for $24 \mathrm{~h}$. The specific peak value of hCQD cannot be found in the spectra of water aliquots, indicating that there was no leaching of hCQDs in water. The absorbance spectrum of hCQD-PU-1, hCQD-PU-10 and hCQD-PU-200 nanocomposites before and after $24 \mathrm{~h}$ immersion in water is presented in (Fig. S1b†). The spectra prove that there is no difference between the nanocomposites before and after dipping the samples in water. Therefore, it can be concluded that leaching of the hCQD from polymer matrix does not occur, when the samples stay immersed in water.

\subsection{Stability of hCQD-PU nanocomposites}

The stability of the gamma-irradiated hCQD-PU nanocomposites was investigated in the presence of the BL and monitored by the UV-vis measurements. According to absorbance spectra presented in Fig. S2, $\uparrow$ we can conclude that the degradation did not occur when the nanocomposites were exposed to the direct $\mathrm{BL}$ on the distance of $10 \mathrm{~cm}$, for $5 \mathrm{~h}$.

\subsection{Contact angle}

The wetting properties of the prepared surfaces were examined by water contact angle (WCA) measurements. As it can be observed from Table S2, $\dagger$ sample hCQD-PU-1 displayed a hydrophobic character. However, the contact angle was decreasing with the increase of the dose of gamma irradiation (Fig. S3 $\dagger$ ). The contact angle decreased from $96.5^{\circ}$ (hCQD-PU-1) to $72.6^{\circ}$ (hCQD-PU-200) which is connected with an increase of surface roughness, calculated from AFM images (Table S2†).

\subsection{Dynamic mechanical analysis (DMA)}

The dynamic mechanical analysis was carried out to determine the effect of gamma irradiation on the integrity of the polymer matrix. As a control sample, we used polyurethane (PU). The DMA results for neat PU matrix and gamma irradiated samples at different doses (1,10 and $200 \mathrm{kGy})$ are presented in Fig. 3. As can be seen from Fig. $3 a$ and b, gamma irradiation did not 
a)

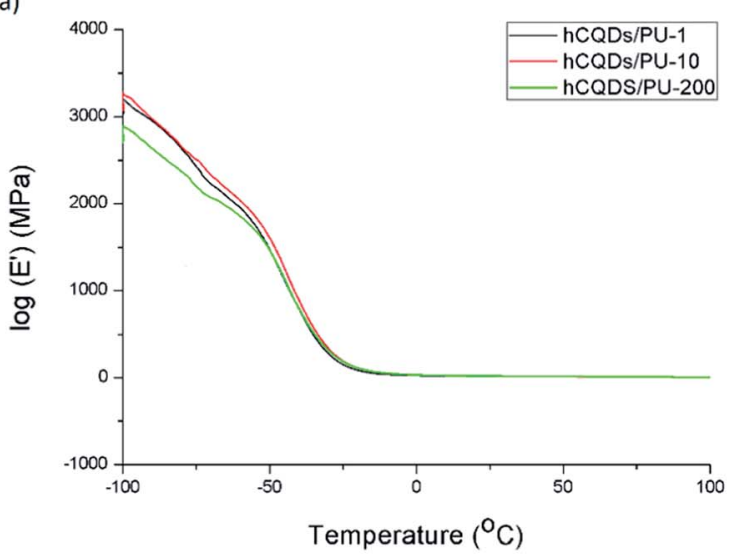

b)

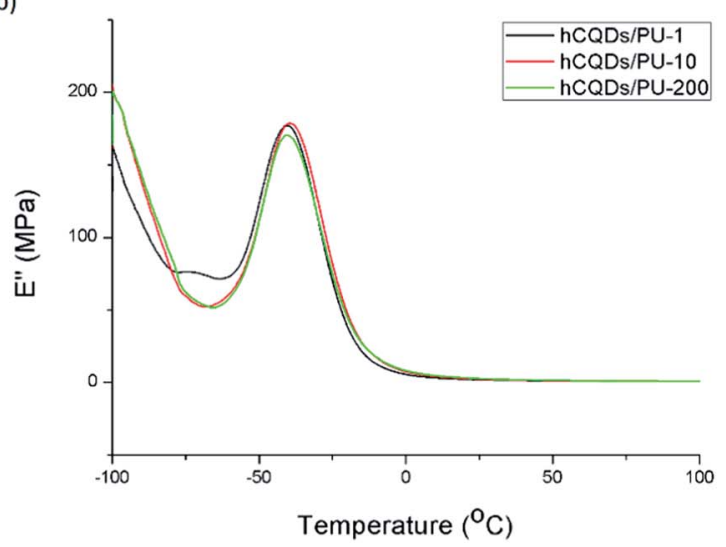

c)

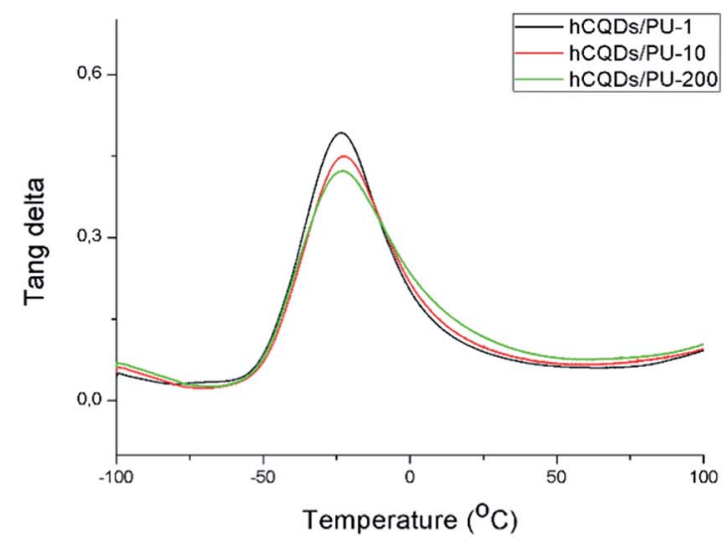

Fig. 3 The dynamic mechanical analysis graphs: (a) loss modules; (b) storage modules; (c) tang delta of hCQD-PU-1, hCQD-PU-10 and hCQD-PU-200 nanocomposite.

significantly affect the structure of the polymer matrix with encapsulated hCQDs. The values of $T_{\mathrm{g}}$ temperatures for neat PU and gamma irradiated samples (hCQD-PU-1, hCQD-PU-10 and hCQD-PU-200) are presented in Table S3. $\dagger$ As can be seen from Table S3, $\dagger$ gamma irradiation contributes to the small decrease of $T_{\mathrm{g}}$ from 0.5 to $1.5{ }^{\circ} \mathrm{C}$ compared to neat PU. According to the results presented in Fig. 3, the peak of $\tan \delta$ curves for all the samples corresponds to a molecular motion of the amorphous region in the semi crystalline hard-segment phase of PU samples. We were not able to detect $T_{\mathrm{g}}$ temperature of softsegment of PU samples due to the lack of the peaks at low temperatures.

\subsection{Atomic force microscopy (AFM)}

AFM was used to analyze the morphology and surface roughness of samples irradiated with different doses of gamma irradiation. From AFM images we can easily observe the difference in the surface morphology of different samples (Fig. 4). This was confirmed by analyzing the average surface roughness value, obtained from several AFM images of each sample (Table S2 $\dagger$ ). The surface roughness significantly increased with the increase of the dose of gamma irradiation.

\subsection{Photoluminescence measurements (PL)}

Photoluminescence of gamma-irradiated hCQD-PU was investigated at different laser excitation wavelengths: from 320 to $480 \mathrm{~nm}$ (Fig. 5). According to Mitra et al. ${ }^{27}$ the hCQDs are probably formed by fragmentation of PF-68 forming the carbon core. Remaining hydroxyl and carbonyl groups on the surface of the hCQDs result in the formation of defect sites ${ }^{28}$ and emissive traps ${ }^{29}$ which contribute to its PL properties. Radiative recombination of excitons trapped within the defects, produces the most intense PL band. Therefore, the PL of gamma modified nanocomposites originates from hCQDs incorporated inside the polymer matrix. ${ }^{22,27}$

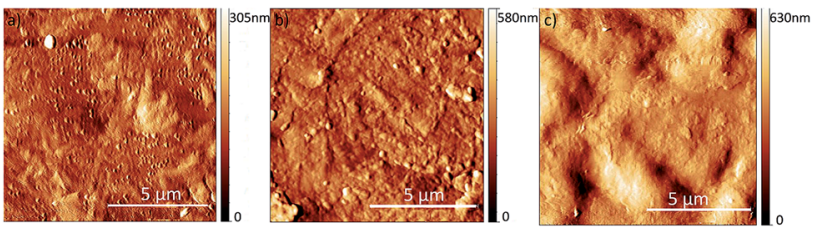

Fig. 4 AFM images of irradiated hCQD-PU, scan size in each image is $10 \times 10 \mu \mathrm{m}$, (a) hCQD-PU-1, (b) hCQD-PU-10, (c) hCQD-PU-200.
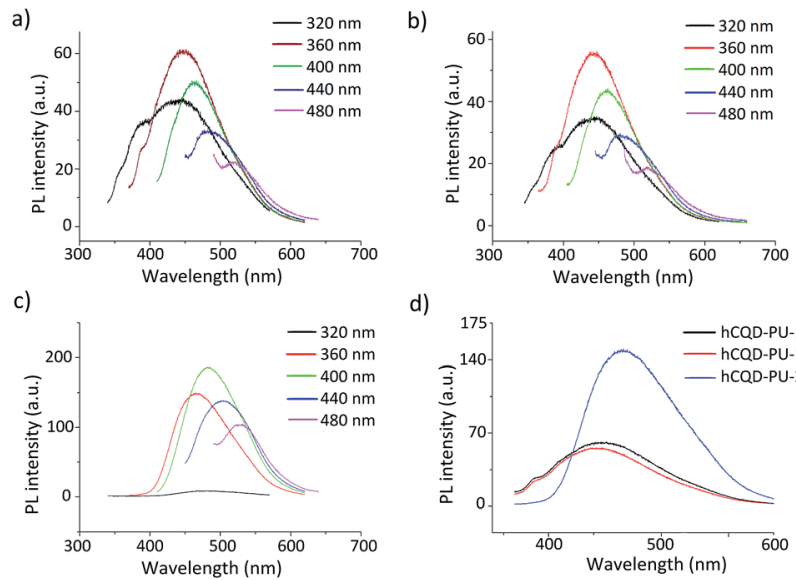

d)

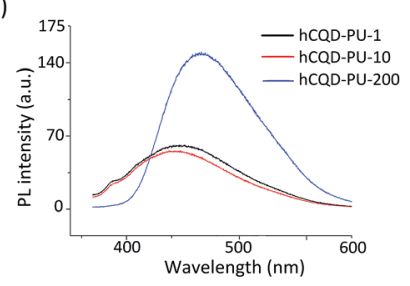

Fig. 5 Photoluminescence spectra of samples (a) hCQD-PU-1, (b) hCQD-PU-10, (c) hCQD-PU-200 at different laser excitation wavelengths and (d) PL spectra of three samples measured at pulsed laser excitation at $360 \mathrm{~nm}$. 
The highest intensities of emission bands were detected at the excitation wavelength of $360 \mathrm{~nm}$ for both hCQD-PU-1 and hCQD-PU-10 samples, while for hCQD-PU-200 sample the highest intensity was measured at $400 \mathrm{~nm}$ excitation wavelength. However, for further examination, we used the excitation wavelength of $360 \mathrm{~nm}$, in order to compare the spectra. As excitation wavelength was increasing, red-shifted emission was noticed, for all the samples. According to some authors, the dominant role in the mechanism of PL emission plays small $\mathrm{sp}^{2}$ isolated within the $\mathrm{sp}^{3}$ carbon network clusters, while the surface states that are oxygen-containing functional groups and defects localized on the edge of $\mathrm{sp}^{2}$ clusters play a supplementary role in the CQDs emission. ${ }^{30,31}$ As can be observed on Fig. 5d, the irradiation dose of $200 \mathrm{kGy}$ has caused significant changes in the PL intensity, which is almost two times more intensive compared to the emission of hCQD-PU-1 and hCQDPU-10 samples. This result is in accordance with the XPS results which proved that the percentage of $\mathrm{sp}^{2}$ bonds is by $10 \%$ higher in hCQD-PU-200 than in hCQD-PU-10 sample. Also, the number of $\mathrm{C}-\mathrm{O}$ groups increased in the hCQD-PU-200 sample. From these results, we can conclude that the content of $\mathrm{sp}^{2}$ bonds and oxygen-containing functional groups play an important role in the mechanism of PL of these samples. Therefore, the role of gamma irradiation was very important here in enhancing the PL. We can also observe (Fig. 5d) that the emission band of hCQD-PU-200 sample is red-shifted compared to hCQD-PU-1 and hCQD-PU-10 samples. The shifts of the emissions spectra of CQDs could be the result of changes in the defect structure, edge configuration, size or shape. ${ }^{19,32,33}$ This may be explained by the decreasing percentage of $\mathrm{sp}^{3}$ and increasing of $\mathrm{sp}^{2}$ bonds, implying the more arranged structure of hCQDs inside the polymer. ${ }^{26}$

\subsection{Electron paramagnetic resonance - EPR}

EPR analysis was performed in the purpose of examining the ability of hCQD-PU nanocomposites to produce singlet oxygen and to understand the role of gamma irradiation in this process. The samples of gamma-irradiated hCQD-PU nanocomposites were recorded with EPR in the presence of 2,2,6,6tetramethylpiperidine (TEMP) which is used as a spin trap agent. The molecules of TEMP selectively react with singlet oxygen $\left({ }^{1} \mathrm{O}_{2}\right)$ and form the stable product 2,2,6,6tetramethylpiperidine-1-oxyl (TEMPO), which shows the characteristic signal. ${ }^{34,35}$ The EPR spectra of TEMP in the dark does not show any signal. With the addition of hCQD-PU in the TEMP solution, even in dark conditions, a very week signal can be noticed, indicating that TEMPO was formed in a small concentration. However, after illumination for $12 \mathrm{~h}$ with $\mathrm{BL}$, significant changes in the EPR spectra can be noticed. The EPR spectra of gamma-irradiated hCQD-PU samples (Fig. 6) consist of three symmetrical signals of Lorentzian shape, from which we can confirm the formation of TEMPO in hCQD-PU nanocomposites. This also confirms their ability to be used as photosensitizers. From the EPR spectra, it can easily be concluded that the sample hCQD-PU-10 has the highest production of singlet oxygen, almost five times stronger than

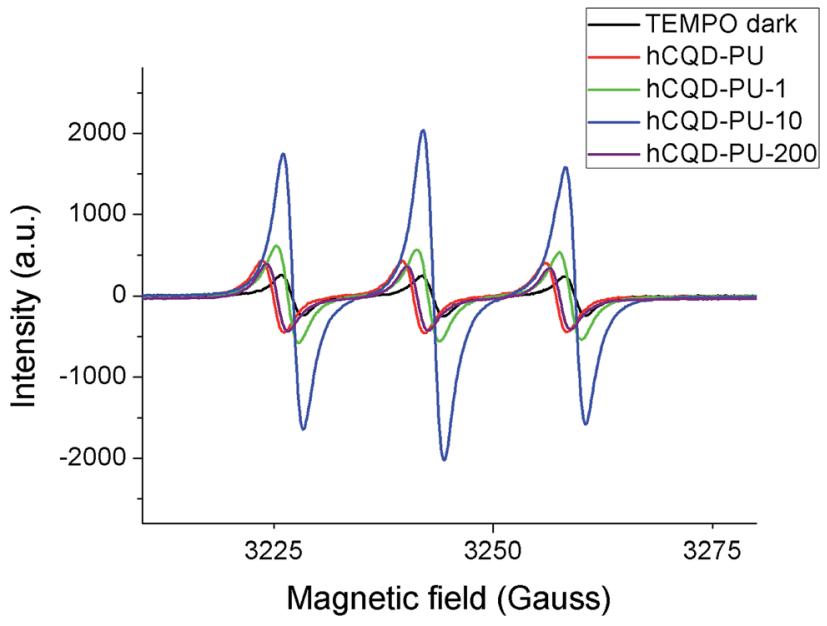

Fig. 6 EPR spectra of hCQD-PU, hCQD-PU-1, hCQD-PU-10 and hCQD-PU-200 composites.

the other two samples. The samples hCQD-PU-200 and hCQDPU-1 are generating less singlet oxygen, due to the stronger presence of hydroxyl groups, which is confirmed by XPS results. The free radical-scavenging capability of GQDs is well known. Chong et al. showed in two separate experiments that GQDs effectively decreased the ESR signal by approximately $56 \%$ due to formation of hydroxyl radicals. ${ }^{36}$ By increasing the dose of gamma irradiation the number of hydroxyl radicals increased and therefore, the scavenging capability of hCQDs-PU samples increased, as well. ${ }^{37}$

\subsection{Photocatalytic properties}

Since the gamma-irradiated hCQD-PU samples showed interesting properties in terms of singlet oxygen production, we decided to explore their ability to degrade organic dyes as a photocatalyst. The CQDs are already proved as an effective visible light driven photocatalyst. ${ }^{38}$ Therefore, in the present section, we explored the photocatalytic properties of the hCQD-PU nanocomposites, and investigated if the gamma irradiation had any impact on their photocatalytic activity. The removal of rose bengal dye by hCQD-PU-1, hCQD-PU-10 and hCQD-PU-200 composites was examined by UV-vis and it can be observed from Fig. 7, where we can see the characteristic absorption peak of RB at $549 \mathrm{~nm}$, which is in accordance with the previous results. ${ }^{39}$ The hCQD-PU-1, hCQD-PU-10 and hCQD-PU-200 samples were dipped in the vials with RB solution and exposed to the BL illumination $(470 \mathrm{~nm})$, in different time intervals. The BL itself had some impact on the RB peak weakening, and it was degraded by $37 \%$ after $3 \mathrm{~h}$. The weakening of the RB peak was proportional to the exposure time to the $\mathrm{BL}$, as well as to the gamma irradiation dose applied to the hCQD-PU nanocomposite samples. From Fig. 7a we can observe that after $1 \mathrm{~h}$ of exposure of hCQD-PU samples and RB solution to the $\mathrm{BL}$ there is no change in the intensity of $\mathrm{RB}$ peak. However, after 3 and $4 \mathrm{~h}$, we can notice a significant decrease of RB peak, as well as the difference in the peak values between each sample. 

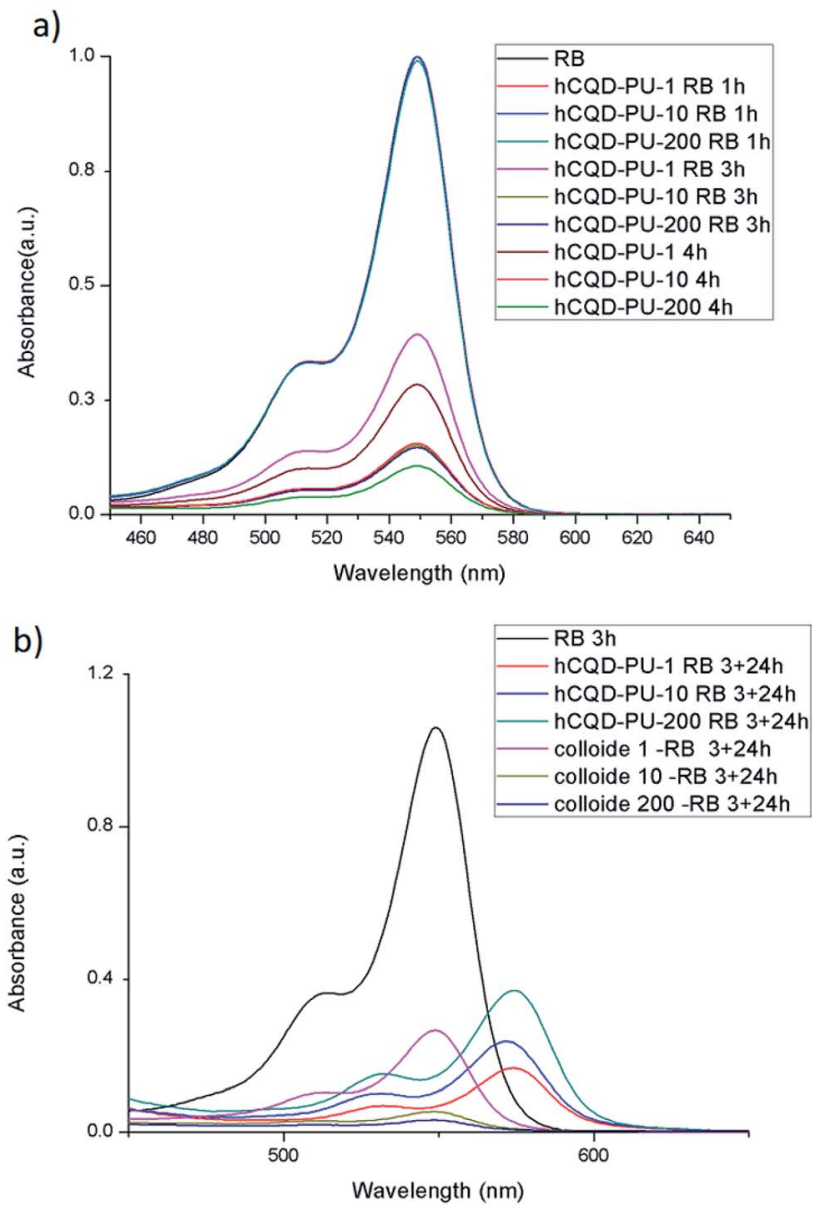

Fig. 7 (a) Degradation of rose bengal after 1, 3 and $4 \mathrm{~h}$ of exposure to the blue lamp in the presence of hCQD-PU samples; (b) absorbance spectra of both, degraded colloid of RB, and composite samples after adsorbing RB after $3 \mathrm{~h}$ of $\mathrm{BL}$ illumination and $24 \mathrm{~h}$ in dark.

We also decided to investigate the impact of the polymer samples on the RB solution in the dark. After $3 \mathrm{~h}$ of BL illumination, we left the polymer samples dipped in the RB
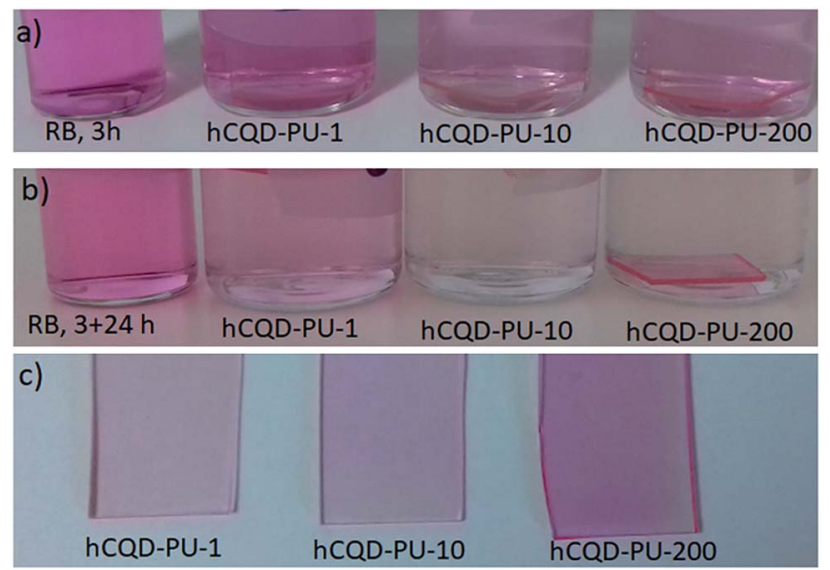

Fig. 8 (a) Water solution of rose bengal $(0.03 \mathrm{mM})$ alone and with samples dipped inside of the solution after (a) $3 \mathrm{~h}$ of exposure to the $\mathrm{BL}$ and (b) $3 \mathrm{~h} \mathrm{BL}$ and $24 \mathrm{~h}$ in dark; (c) samples hCQD-PU after $24 \mathrm{~h}$ in the RB solution. solution for $24 \mathrm{~h}$ in dark. The UV-vis measurements of colloids and polymers were performed the next day. From (Fig. 8) we can see that the RB solution as a control sample remained the same, but the RB solution in which the samples were dipped lost its color completely, while the typical peak at $549 \mathrm{~nm}$ disappeared. On the other hand, we also observed the UV-vis absorption of the polymer nanocomposites before and after dipping in the RB solution (Fig. S4a $\uparrow$ ), where we could observe that the RB peak appeared in the nanocomposites and it was blue shifted to $574 \mathrm{~nm}$ value. Additionally, we could observe that the polymer nanocomposites themselves changed their color to pink, implying that the RB was absorbed into the polymer matrix together with hCQDs (Fig. 8).

The absorbance of polymer nanocomposite samples confirmed that hCQD-PU-200 sample had absorbed the highest concentration of RB, and hCQD-PU-1 the lowest, which is in accordance with the results obtained from absorbance measurements of the RB solution after dipping the samples (Table 1). This result is also in accordance with the surface morphology and surface roughness measurements, obtained by AFM, where it was shown that hCQD-PU200 sample had less hydrophobic character than the other two samples. From Fig. S4a $\uparrow$ we can observe the degradation of the RB peak in the colloid where the samples were immersed. Additionally, we can notice the shift of the RB peak which was detected inside of the hCQD-PU nanocomposite samples (Fig. 7b).

By analysing the absorbance spectra (Fig. S4 $\uparrow$ ) and statistic data presented in Table 1, we can conclude that the overall efficiency of dye degradation is the highest in the hCQD-PU-200 sample $(97 \%)$. This value was calculated from the UV-vis spectra, where the peak of the RB solution after $3 \mathrm{~h}$ of BL illumination was used as a reference $(100 \%)$. However, the degree of degradation of $\mathrm{RB}$ dye by photosensitization was the strongest in hCQD-PU-10 sample (72\%), and we calculated this result after we measured the percentage of adsorbed RB inside the polymer nanocomposites.

This result is in accordance with EPR measurements, which showed that the hCQD-PU-10 nanocomposite had the highest singlet oxygen production. We believe that the mechanism of the dye removal here is a combination of two different mechanisms. First, the photocatalytic degradation by singlet oxygen production, and second is the absorption by the polymer matrix. The experiments showed that the best absorption was in the sample hCQD-PU-200, which was in accordance with the contact angle and surface roughness measurements. This was confirmed by the absorbance spectra of all the three polymer samples after they remained in the RB solution for $24 \mathrm{~h}$. The sample hCQD-PU-200 was able to absorb the highest percentage of RB dye (37\%), while the smallest concentration was absorbed by hCQD-PU-1 nanocomposite (17\%). The overall efficiency of the RB dye removal, as the sum of the two combined mechanisms, was the weakest in hCQD-PU and hCQD-PU-1 samples ( $72 \%$ and $74 \%$ ) while in the hCQD-PU-10 and hCQD-PU-200 nanocomposites it was significantly higher (95\% and 97\%) respectively. 


\section{Conclusion}

Here we demonstrated a successful and cost-effective preparation method of hCQD-PU nanocomposites and the influence of gamma irradiation modification method on their physical and chemical properties. These modified nanocomposites were explored for the possible application as catalysts in the photocatalytic degradation process of rose bengal organic dye. The detailed characterization of gamma-modified hCQD-PU nanocomposites showed certain changes in the surface roughness and contact angle, but the mechanical stability of nanocomposites was not significantly influenced by the gammairradiation. Additionally, we concluded that the photoluminescence was present in all the samples, but gamma irradiation had an influence on their photoluminescent properties and it was increasing with the increase of the dose of gamma irradiation. Gamma irradiation had an impact on the singlet oxygen production as well, and the analysis proved that the highest singlet oxygen production was found in the nanocomposite irradiated with the dose of $10 \mathrm{kGy}$. Also, the percentage of $\mathrm{sp}^{2}$ groups significantly increased with the increase of the irradiation dose. Therefore, we can conclude that gamma irradiation did have a significant influence on the chemical properties of nanocomposites. The possible use of the hCQD-PU nanocomposites in the degradation of rose bengal dye was explored. By combining two different mechanisms (photocatalytic degradation by singlet oxygen production, and the absorption by the polymer matrix) these nanocomposites showed excellent dye removal efficiency, reaching 97\%. The gamma irradiated hCQD-PU nanocomposites did not show leaching after $24 \mathrm{~h}$ of immersion in water, which is an important feature for their possible use in water purification systems. These nanocomposites have the potential in the removal of other pollutants from water, as well.

\section{Conflicts of interest}

There are no conflicts to declare.

\section{Acknowledgements}

Authors thank for support to the Ministry of Education, Science and Technological Development of the Republic of Serbia via project no. 172003. Author Zoran Markovic thanks for funding by a STSM grant from the COST Action CA16217 "ENIUS" and funded by COST (European Cooperation in Science and Technology).

\section{References}

1 F. M. D. Chequer, V. de P. Venâncio, M. de L. P. Bianchi and L. M. G. Antunes, Food Chem. Toxicol., 2012, 50, 3447-3451.

2 J. Kaur and S. Singhal, Phys. Rev. B: Condens. Matter Mater. Phys., 2014, 450, 49-53.

3 I. Ali, Chem. Rev., 2012, 112, 5073-5091.

4 B. Tanhaei, A. Ayati, M. Lahtinen and M. Sillanpää, Chem. Eng. J., 2015, 259, 1-10. 
5 J. Y. Bottero, J. Rose and M. R. Wiesner, Integr. Environ. Assess. Manage., 2006, 2, 391-395.

6 Y. Zhang, B. Wu, H. Xu, H. Liu, M. Wang, Y. He and B. Pan, NanoImpact, 2016, 3-4, 22-39.

7 O. J. Hao, H. Kim and P.-C. Chiang, Crit. Rev. Environ. Sci. Technol., 2000, 30, 449-505.

8 M. N. Chong, B. Jin, C. W. K. Chow and C. Saint, Water Res., 2010, 44, 2997-3027.

9 S. De Gisi, G. Lofrano, M. Grassi and M. Notarnicola, Sustainable Mater. Technol., 2016, 9, 10-40.

10 J. Yin and B. Deng, J. Membr. Sci., 2015, 479, 256-275.

11 S. J. Tesh and T. B. Scott, Adv. Mater., 2014, 26, 6056-6068.

12 S. Y. Lim, W. Shen and Z. Gao, Chem. Soc. Rev., 2015, 44, 362381.

13 H. Li, Z. Kang, Y. Liu and S.-T. Lee, J. Mater. Chem., 2012, 22, 24230.

14 S. N. Baker and G. A. Baker, Angew. Chem., Int. Ed., 2010, 49, 6726-6744.

15 D. E. J. G. J. Dolmans, D. Fukumura and R. K. Jain, Nat. Rev. Cancer, 2003, 3, 380-387.

16 Y. Yang, X. Wang, G. Liao, X. Liu, Q. Chen, H. Li, L. Lu, P. Zhao and Z. Yu, J. Colloid Interface Sci., 2018, 509, 515-521.

17 P. Zhao, Q. Xu, J. Tao, Z. Jin, Y. Pan, C. Yu and Z. Yu, Wiley Interdiscip. Rev.: Nanomed. Nanobiotechnol., 2018, 10, e1483.

18 Q. Xu, T. Kuang, Y. Liu, L. Cai, X. Peng, T. Sreenivasan Sreeprasad, P. Zhao, Z. Yu and N. Li, J. Mater. Chem. B, 2016, 4, 7204-7219.

19 J. Ge, M. Lan, B. Zhou, W. Liu, L. Guo, H. Wang, Q. Jia, G. Niu, X. Huang, H. Zhou, X. Meng, P. Wang, C. S. Lee, W. Zhang and X. Han, Nat. Commun., 2014, 5, 1-8.

20 L. Montanari, M. Costantini, E. C. Signoretti, L. Valvo, M. Santucci, M. Bartolomei, P. Fattibene, S. Onori, A. Faucitano, B. Conti and I. Genta, J. Controlled Release, 1998, 56, 219-229.

21 S. P. Jovanović, Z. Syrgiannis, Z. M. Marković, A. Bonasera, D. P. Kepić, M. D. Budimir, D. D. Milivojević, V. D. Spasojević, M. D. Dramićanin, V. B. Pavlović and B. M. Todorović Marković, ACS Appl. Mater. Interfaces, 2015, 7(46), 25865-25874.

22 M. Kovacova, Z. M. Markovic, P. Humpolicek, M. Micusik, H. Švajdlenkova, A. Kleinova, M. Danko, P. Kubát, J. Vajdak, Z. Capakova, M. Lehocký, L. Munster, B. M. Todorović Marković and Z. Spitalsky, ACS Biomater. Sci. Eng., 2018, 4(12), 3983-3993.
23 N. K. Stanković, M. Bodik, P. Šiffalovič, M. Kotlar, M. Mičušik, Z. Špitalsky, M. Danko, D. D. Milivojević, A. Kleinova, P. Kubat, Z. Capakova, P. Humpoliček, M. Lehocky, B. M. Todorović Marković and Z. M. Marković, ACS Sustainable Chem. Eng., 2018, 6, 4154-4163.

24 D. Nečas and P. Klapetek, Open Phys., 2012, 10, 181-188.

25 W. Kwon and S.-W. Rhee, Chem. Commun., 2012, 48, 5256.

26 S. H. Al-Harthi, M. Elzain, M. Al-Barwani, A. Kora'a, T. Hysen,

M. T. Z. Myint and M. R. Anantharaman, Nanoscale Res. Lett., 2012, 7, 466.

27 S. Mitra, S. Chandra, T. Kundu, R. Banerjee, P. Pramanik and A. Goswami, RSC Adv., 2012, 2, 12129-12131.

28 Y. Yang, J. Cui, M. Zheng, C. Hu, S. Tan, Y. Xiao, Q. Yang and Y. Liu, Chem. Commun., 2012, 48, 380-382.

29 Y. P. Sun, B. Zhou, Y. Lin, W. Wang, K. A. Shiral Fernando, P. Pathak, M. J. Meziani, B. A. Harruff, X. Wang, H. Wang, P. G. Luo, H. Yang, M. E. Kose, B. Chen, L. M. Veca and S.-Y. Xie, J. Am. Chem. Soc., 2006, 128, 7756.

30 H. Yan, C. He, X. Li and T. Zhao, Diamond Relat. Mater., 2018, 87, 233-241.

31 G. Eda, Y.-Y. Lin, C. Mattevi, H. Yamaguchi, H.-A. Chen, I.-S. Chen, C.-W. Chen and M. Chhowalla, Adv. Mater., 2010, 22, 505-509.

32 S. P. Jovanović, Z. M. Marković, Z. Syrgiannis, M. D. Dramićanin, F. Arcudi, V. L. Parola, M. D. Budimir and B. M. T. Marković, Mater. Res. Bull., 2017, 93, 183.

33 X. Yan, B. Li, X. Cui, Q. Wei, K. Tajima and L. Li, J. Phys. Chem. Lett., 2011, 2, 1119-1124.

34 Y. Lion, M. Delmelle and A. Van De Vorst, Nature, 1976, 263, 442-443.

35 J. Moan and E. Wold, Nature, 1979, 279, 450-451.

36 Y. Chong, C. Ge, G. Fang, X. Tian, X. Ma, T. Wen, W. G. Wamer, C. Chen, Z. Chai and J.-J. Yin, ACS Nano, 2016, 10, 8690-8699.

37 S. P. Jovanović, Z. Syrgiannis, Z. M. Marković, A. Bonasera, D. P. Kepić, M. D. Budimir, D. D. Milivojević, V. D. Spasojević, M. D. Dramićanin, V. B. Pavlović and B. M. Todorović Marković, ACS Appl. Mater. Interfaces, 2015, 7, 25865-25874.

38 H. Li, R. Liu, W. Kong, J. Liu, Y. Liu, L. Zhou, X. Zhang, S.-T. Lee and Z. Kang, Nanoscale, 2014, 6, 867-873.

39 R. W. Sabnis, Handbook of Biological Dyes and Stains, Synthesis and Industrial Applications, 2010. 